PHYSICAL REVIEW D 96, 089903(E) (2017)

\title{
Publisher's Note: Constraining a dark matter and dark energy interaction scenario with a dynamical equation of state [Phys. Rev. D 95, 123527 (2017)]
}

Weiqiang Yang, Narayan Banerjee, and Supriya Pan (Received 16 October 2017; published 30 October 2017)

DOI: 10.1103/PhysRevD.96.089903

This paper was published online on 20 June 2017 with errors in Eqs. (7), (9), and (16). Equation (7) should read as

$$
\rho_{D E}=\frac{(1+z)^{3}}{f(z)}\left[\rho_{D E, 0} f(0)+\delta \rho_{d m, 0} \int_{0}^{z}(1+z)^{-1-\delta} f(z) d z\right] .
$$

Equation (9) should read as

$$
\begin{aligned}
\left(\frac{H}{H_{0}}\right)^{2}= & \Omega_{r 0}(1+z)^{4}+\Omega_{b 0}(1+z)^{3}+\Omega_{d m, 0}(1+z)^{3-\delta} \\
& +\frac{(1+z)^{3}}{f(z)}\left[\Omega_{D E, 0} f(0)+\delta \Omega_{d m, 0} \int_{0}^{z}(1+z)^{-1-\delta} f(z) d z\right] .
\end{aligned}
$$

Equation (16) should read as

$$
\begin{aligned}
\left(\frac{H}{H_{0}}\right)^{2}= & \Omega_{r 0}(1+z)^{4}+\Omega_{b 0}(1+z)^{3}+\Omega_{d m, 0}(1+z)^{3+3 \eta} \\
& +\Omega_{D E, 0}(1+z)^{3\left(1+w_{0}+\frac{w_{\beta}}{\beta}\right)} \exp \left(\frac{3 w_{\beta}}{\beta^{2}}\left\{(1+z)^{-\beta}-1\right\}\right) .
\end{aligned}
$$

The authors thank Somasri Sen for finding the misprints. The paper has been corrected as of 17 October 2017. The equations are correct in the printed version of the journal. 